\title{
A Quality Conundrum: Well Done but Not Enough
}

Chayan Chakraborti, mD

Section of General Internal Medicine and Geriatrics, Tulane University Health Sciences Center,

New Orleans, Louisiana.

Prado et al.'s ${ }^{1}$ insightful analysis on a rapid response system failure draws attention to "afferent limb system failures" of medical emergency teams (METs). The article also serves to highlight several key quality improvement (QI) educational points. The authors demonstrate a thorough grasp of the literature concerning METs. The case description reveals a detailed investigation that is thorough enough to create a timeline of events. I applaud the literature review and construction of a timeline, as these represent the first several steps of a root-cause analysis-but they are somewhat insufficient. More work can be done here.

Extending their line of inquiry may uncover specific system factors involved in the afferent limb failure. To further the analysis, careful interviews of all involved personnel (including patients, family members, and nurses) may help identify the factors that compromise afferent limbs of METs and thereby make necessary improvements, as in the innovative Josie King Safety Program at Johns Hopkins Hospital (Baltimore, MD). Prado et al. ${ }^{1}$ are extremely fortunate in that their institution has a monitoring system in place to track MET activations. A more ambitious, though potentially more fruitful project, would be to, examine previous afferent limb failures in an effort to identify systems factors that are more generalizable to other institutions.

The difficulties in obtaining data are 2-fold: first in gathering the data, and second in extending the data beyond one's own institution. The very nature of QI data, eg, data that are locally obtained and relevant to a particular institution, hinders its generalizability. However, afferent limb failures are real and perhaps ubiquitous. ${ }^{2,3}$ The challenge then, is to develop strategies that can improve the functioning of METs (both afferent and efferent limbs) regardless of the institution.

As afferent limbs of METs have been identified as a priority for future attention for the greatest benefit, ${ }^{2,4}$ the process of analyzing root-causes of systems failures seems to be analogous to identifying risk factors for a novel disease.
Once identified, the appropriate risk-factor modifications can be undertaken. Only by careful examination of the data can true, relevant factors be identified. For this reason, I feel that Prado et al.'s ${ }^{1}$ excellent work should be expanded upon and replicated in other institutions.

Should these types of QI projects become more amenable to extrapolation to other institutions, a predominant reporting format may be needed. The Standards for Quality Improvement Reporting Excellence (SQUIRE) guideline http://www.squire-statement.org/guidelines for reporting a QI project is an example of 1 such format. This is particularly relevant as hospitalists are increasingly encouraged to be more productive beyond clinical excellence. QI represents a clear avenue for this productivity, though a recent commentary suggests that most QI projects are not published or publishable. ${ }^{5}$ On the other hand, the authors of that article do advocate the development of quality dossiers analogous to educators portfolios that can be useful for promotions. ${ }^{5}$ At least 1 organization has assembled a framework in the quality portfolio in anticipation of the utility of quality dossiers. ${ }^{6}$

\section{References}

1. Prado R, Albert RK, Mehler PS, Chu ES. Rapid response: a quality improvement conundrum. J Hosp Med. 2009;4(4):255-257.

2. Ranji SR, Auerbach AD, Hurd CJ, O'Rourke K, Shojania KG. Effects of rapid response systems on clinical outcomes: systematic review and meta-analysis. J Hosp Med. 2007;2(6):422-432.

3. McGaughey J, Alderdice F, Fowler R, et al. Outreach and early warning systems (EWS) for the prevention of intensive care admission and death of critically ill adult patients on general hospital wards. Cochrane Database Syst Rev 2007(3):CD005529.

4. Hillman $\mathrm{K}$, Chen J, Cretikos $\mathrm{M}$, et al. Introduction of the medical emergency team (MET) system: a cluster-randomised controlled trial. Lancet. 2005;365(9477):2091-2097.

5. Shojania KG, Levinson W. Clinicians in quality improvement: a new career pathway in academic medicine. JAMA. 2009;301(7):766-768.

6. SGIM. Quality Portfolio Introduction. Available at: http://www.sgim.org/ index.cfm?pageId=846. Accessed September 2009. 